\title{
WEIGHTED FRACTIONAL CHAIN RULE AND NONLINEAR WAVE EQUATIONS WITH MINIMAL REGULARITY
}

\author{
KUNIO HIDANO, JIN-CHENG JIANG, SANGHYUK LEE, AND CHENGBO WANG
}

\begin{abstract}
We consider the local well-posedness for 3-D quadratic semi-linear wave equations with radial data:

$$
\begin{gathered}
\square u=a\left|\partial_{t} u\right|^{2}+b\left|\nabla_{x} u\right|^{2}, \\
u(0, x)=u_{0}(x) \in H_{\mathrm{rad}}^{s}, \partial_{t} u(0, x)=u_{1}(x) \in H_{\mathrm{rad}}^{s-1} .
\end{gathered}
$$

It has been known that the problem is well-posed for $s \geq 2$ and ill-posed for $s<3 / 2$. In this paper, we prove unconditional well-posedness up to the scaling invariant regularity, that is to say, for $s>3 / 2$ and thus fill the gap which was left open for many years. For the purpose, we also obtain a weighted fractional chain rule, which is of independent interest. Our method here also works for a class of nonlinear wave equations with general power type nonlinearities which contain the space-time derivatives of the unknown functions. In particular, we prove the Glassey conjecture in the radial case, with minimal regularity assumption.
\end{abstract}

\section{INTRODUCTION}

In this paper, we are interested in the problem of local well-posedness with minimal regularity for 3-D quadratic semi-linear wave equations with radial data,

$$
\begin{gathered}
\square u=a\left|\partial_{t} u\right|^{2}+b\left|\nabla_{x} u\right|^{2}, \\
u(0, x)=u_{0}(x) \in H_{\mathrm{rad}}^{s}, \partial_{t} u(0, x)=u_{1}(x) \in H_{\mathrm{rad}}^{s-1},
\end{gathered}
$$

where $(t, x) \in \mathbb{R}_{+} \times \mathbb{R}^{3},(a, b) \in \mathbb{R}^{2} \backslash\{(0,0)\}, \square=\partial_{t}^{2}-\Delta, \partial=\left(\partial_{t}, \nabla_{x}\right)$. Here $H_{\text {rad }}^{s}$ denotes the space of spherically symmetric functions lying in the usual Sobolev space $H^{s}$. Notice that $s_{c}=3 / 2$ is the regularity for the problem to be scale invariant in the homogeneous Sobolev space $\dot{H}^{s}$, and it is well known that the problem is ill-posed in $H^{s}$ and $H_{\mathrm{rad}}^{s}$ for $s<s_{c}$, see Lindblad [23]. The equation (1.1) serves as a simplified model for many equations which are important in mathematical physics, such as wave maps and Einstein equations. The problem of local wellposedness with minimal regularity assumption has been extensively investigated and well-understood.

1.1. Quadratic semi-linear wave equation. In [19], by using bilinear estimates, together with standard energy-type estimates, Klainerman and Machedon proved the local well-posedness for the problem (1.1) with $a+b=0$ (thus, satisfying the null condition) in $H^{2}$, which was later improved to $H^{s}$ for $s>s_{c}$, see [20], [21], [22] and references therein. Moreover, even without the null condition, their bilinear

Date: October 11, 2018.

2010 Mathematics Subject Classification. 35L70, 35L15, 42B25, 42B37.

Key words and phrases. Glassey conjecture, fractional chain rule, nonlinear wave equations, generalized Strichartz estimates, unconditional uniqueness. 
estimate holds if initial data and forcing terms are radially symmetric in the spatial variables. It yields local well-posedness in $H_{\text {rad }}^{2}$ for the problem (1.1), and they conjectured that the problem is ill posed in $H^{2}$ in general. Later, Ponce-Sideris [31] proved local well-posedness in $H^{s}$ for any $s>2$, as well as the radial case $H_{\text {rad }}^{2}$, by applying the Strichartz estimates. See also Tataru [39], Smith-Tataru [35] and references therein for related results on local well-posedness for the same problem as well as the quasilinear wave equation $\square_{g(u)} u=Q(\partial u, \partial u)$, in general spatial dimensions. In [23], [24], Lindblad proved that the problem is generally ill-posed in $H^{s}$ for any $s \leq 2$, which verified the conjecture of Klainerman-Machedon [19]. Thus, in general cases the local well-posedness problem looks almost completely understood in terms of regularity of the initial data. However, if we focus on the radial data, the current state is not so satisfactory. To be precise, the results of Lindblad [23], [24] do not apply to the radial solutions, so we only know that the problem is ill-posed in $H_{\mathrm{rad}}^{s}$ with $s<s_{c}=3 / 2$ (see Remark 1.2). On the side of well-posedness, from [19] the problem (1.1) is locally well-posed in $H_{\mathrm{rad}}^{2}$. This means, in contrast to the general cases, that for the radial case there is still a gap between ill-posedness and well-posedness results, precisely for (1.1) in $H_{\text {rad }}^{s}$ with $s \in[3 / 2,2)$.

In the following which is our main result we prove that $s>s_{c}$ is sufficient for (1.1) to be locally well-posed so that we fill the gap except the case $s=s_{c}$. Moreover, we show that the local solutions could be extended to almost global solutions in $H_{\mathrm{rad}}^{s}$, when the initial data are small. Since the result for $s \geq 2$ was already proved in the aforementioned previous works, we restrict ourselves to the case $s \in\left(s_{c}, 2\right)=(3 / 2,2)$.

Theorem 1.1. The problem (1.1) is unconditionally well-posed in $H_{\mathrm{rad}}^{s}$ with $s \in$ $(3 / 2,2)$. More precisely, for any radial data $\left(u_{0}, u_{1}\right) \in H_{\mathrm{rad}}^{s} \times H_{\mathrm{rad}}^{s-1}$, there exists $T \in(0, \infty)$, which depends only on the norm of $\left(u_{0}, u_{1}\right)$, such that the problem (1.1) admits a unique, radially symmetric solution $u \in L^{\infty}\left([0, T] ; H^{s}\left(\mathbb{R}^{3}\right)\right) \cap$ $\operatorname{Lip}\left([0, T] ; H^{s-1}\left(\mathbb{R}^{3}\right)\right)$. Moreover, $u \in C\left([0, T] ; H^{s}\right) \cap C^{1}\left([0, T] ; H^{s-1}\right)$,

$$
r^{-1 / 2+\delta}\langle r\rangle^{-\delta} \partial u \in L^{2}\left([0, T] \times \mathbb{R}^{3}\right), 2 \delta=s-\frac{3}{2},
$$

and there exists $c>0$, such that we can choose $T=T_{\varepsilon}$, where

$$
T_{\varepsilon}= \begin{cases}\exp \left(c \varepsilon^{-1}\right), & \varepsilon<1 \\ c \varepsilon^{-\frac{1}{s-3 / 2}}, & \varepsilon \geq 1\end{cases}
$$

with

$$
\varepsilon=\|\partial u(0)\|_{\dot{H}^{s-1}}+\|\partial u(0)\|_{\dot{H}^{s-1}}^{1 / 2}\|\partial u(0)\|_{\dot{H}^{2-s}}^{1 / 2} .
$$

We now make several remarks concerning Theorem 1.1.

Remark 1.1. It is natural to expect that solutions exist for longer time as the initial data get smaller but it is another matter to obtain precise quantification of the lifespan depending on the size of the initial data. For the problem (1.1) with generic compactly supported smooth data of size $\varepsilon$, it is known that the lifespan $T_{\varepsilon}$ of the classical solution satisfies

$$
\ln T_{\varepsilon} \simeq \varepsilon^{-1},
$$

except the case when the null conditions are satisfied (in which case we have small data global existence), see John [16], John-Klainerman [15], Rammaha [32], Zhou 
[44]. For $H_{\mathrm{rad}}^{2}$ data Hidano-Yokoyama [12] proved almost global existence, i.e., $T_{\varepsilon} \geq \exp \left(c \varepsilon^{-1}\right)$, of the Klainerman-Machedon radial solutions for small $H_{\mathrm{rad}}^{2}$ data by using a variant of the KSS estimate [18]. See Hidano-Wang-Yokoyama [11] for a related result in the quasilinear case. See also Sterbenz [38] and references therein for small data global well-posedness with low regularity, for (1.1) with spatial dimension $n \geq 4$.

Remark 1.2. The regularity assumption in Theorem 1.1, $s>s_{c}=3 / 2$, is optimal, in the sense that the same conclusion fails for $s=s_{c}$ in general. More precisely, for (1.1) with $a=1$, there exists a family of compactly supported, smooth, radially symmetric functions $g_{\lambda}$, which are uniformly bounded in $H_{\mathrm{rad}}^{1 / 2}$, such that the lifespan of the corresponding solution $u_{\lambda}$ in $H_{\mathrm{rad}}^{s}$, with data $u(0)=0, u_{t}(0)=g_{\lambda}$, goes to zero as $\lambda$ goes to $\infty$. See the proof of Fang-Wang [3, Theorem 1.2] together with an explicit ODE solution $u_{\lambda}(x, t)=-\ln (1-t / \lambda)$ instead of (3.3) there.

Remark 1.3. The unconditional uniqueness for the problem (1.1) has been obtained in $H^{s}$ with $s>2$ in Planchon [30]. See also Zhou [43] for a related $H^{2}$ result under the null condition.

1.2. Weighted fractional chain rule. The well-posedness results in [19], [31] are based on bilinear and Strichartz estimates. As is clear, such approach doesn't seem to work for our problems. Instead, for the proof of Theorem 1.1, we make use of the Morawetz type estimates for the wave equations (also known as local energy estimates and KSS type estimates in the literature) and a weighted fractional chain rule which is of independent interest. As far as we are aware, this type of chain rule hasn't appeared before. As Morawetz type estimates could be proved by multiplier methods (for example, see $[37,27]$ ) our proof of Theorem 1.1 provides a physical space approach for the nonlinear wave equations with low regularity. We believe that our approach will be useful for studies in related nonlinear problems.

As for the weight functions, we use Muckenhoupt's $A_{p}$ class. Let us recall the definition of $A_{p}$ weights:

$$
w \in A_{1} \Leftrightarrow \mathcal{M} w(x) \leq C w(x), \text { a.e. } x \in \mathbb{R}^{n},
$$

and, for $1<p<\infty, w \in A_{p}$ if and only if $w \geq 0$ and

$$
\left(\int_{Q} w(x) d x\right)\left(\int_{Q} w^{1-p^{\prime}}(x) d x\right)^{p-1} \leq C|Q|^{p}, \quad \forall \text { cubes } Q .
$$

Here $\mathcal{M}$ is the Hardy-Littlewood Maximal function which is defined by $\mathcal{M} w(x)=$ $\sup _{r>0} r^{-n} \int_{B_{r}(x)}|w(y)| d y$.

Theorem 1.2 (Weighted fractional chain rule). Let $s \in(0,1), q, q_{1}, q_{2} \in(1, \infty)$ with $\frac{1}{q}=\frac{1}{q_{1}}+\frac{1}{q_{2}}$. Assume $F: \mathbb{R}^{k} \rightarrow \mathbb{R}^{l}$ is a $C^{1}$ map, satisfying $F(0)=0$ and

$$
\left|F^{\prime}(\tau v+(1-\tau) w)\right| \leq \mu(\tau)|G(v)+G(w)|,
$$

with $G \geq 0$ and $\mu \in L^{1}([0,1])$. If $\left(w_{1} w_{2}\right)^{q} \in A_{q}, w_{1}^{q_{1}} \in A_{q_{1}}, w_{2}^{q_{2}} \in A_{q_{2}}$, then

$$
\left\|w_{1} w_{2} D^{s} F(u)\right\|_{L^{q}} \lesssim\left\|w_{1} D^{s} u\right\|_{L^{q_{1}}}\left\|w_{2} G(u)\right\|_{L^{q_{2}}},
$$

where $D=\sqrt{-\Delta}$. In addition, when $q_{2}=\infty$ and $q \in(1, \infty)$, if $w_{1}^{q},\left(w_{1} w_{2}\right)^{q} \in A_{q}$ and $w_{2}^{-1} \in A_{1}$, we have

$$
\left\|w_{1} w_{2} D^{s} F(u)\right\|_{L^{q}} \lesssim\left\|w_{1} D^{s} u\right\|_{L^{q}}\left\|w_{2} G(u)\right\|_{L^{\infty}} .
$$


The fractional chain rule with $w_{1}=w_{2} \equiv 1$ (without weights) was proven by Christ-Weinstein [2], Staffilani [36], Kato [17]. See also Taylor [40, Chapter 2 Proposition 5.1]. Our proof of Theorem 1.2 basically follows the same line of agrument as in Taylor [40] but we also need new ingredients such as the weighted estimates for Calderón-Zygmund, Littlewood-Paley operators and weighted vector valued inequality for Hardy-Littlewood maximal functions (see Andersen-John [1]). We note the further potential of the fractional chain rule in Theorem 1.2 and the characterization of our weight $w(x)=r^{-1+2 \delta}\langle r\rangle^{-2 \delta-2 \delta_{1}}$ in terms of the Muckenhoupt $A_{1}$ class (see Lemma 2.5 below). They also play an important role in the study of well-posedness of the Cauchy problem for the nonlinear half wave equation and the nonlinear elastic wave equation with low-regularity data. See Hidano-Wang [9], Hidano-Zha [13].

1.3. General power type nonlinearities. We also apply our method to a class of nonlinear wave equations with general power type nonlinearities in which the spacetime derivatives of the unknown functions also appear. More precisely, let $n \geq 2$, $p>1$, and consider the following nonlinear wave equation, with $(t, x) \in \mathbb{R}_{+} \times \mathbb{R}^{n}$,

$$
\begin{gathered}
\square u=a\left|\partial_{t} u\right|^{p}+b|\nabla u|^{p}, \\
u(0, x)=u_{0}(x) \in H_{\mathrm{rad}}^{s}, \partial_{t} u(0, x)=u_{1}(x) \in H_{\mathrm{rad}}^{s-1} .
\end{gathered}
$$

For this problem, $s_{c}=(n+2) / 2-1 /(p-1)$ is the scaling-critical Sobolev regularity, which is a lower bound for the problem to be locally well-posed in $H^{s}$, and also $H_{\mathrm{rad}}^{s}$. In addition, $s_{l}=(n+5) / 4$ is a lower bound for the problem to be locally well-posed in $H^{s}$. See Fang-Wang [3] for these ill-posed results when $n \geq 3^{1}$. If the nonlinearity is sufficiently smooth, the problem is locally well-posed in $H^{s}$, for $s \geq s_{c}$ and $s>\max \left(s_{l},(n+1) / 2\right)$. This can be shown by using Strichartz estimates, see, e.g., Fang-Wang [3]. Moreover, when the initial data are radial, improved Strichartz type estimates under radial symmetry can be further exploited to prove local well-posedness in $H_{\mathrm{rad}}^{s}$ for $s \geq \max \left(s_{c},(n+1) / 2\right)$ and $s>3 / 2$ (see, Fang-Wang [4], [6]).

Concerning long time existence with small data, the problem is related to the so-called Glassey conjecture (see Glassey's MR review of the paper of Sideris [33]), which states that the critical power for (1.6) to admit global small solutions is

$$
p_{c}(n) \equiv 1+\frac{2}{n-1} \text {. }
$$

The global existence for $p>p_{c}(n)$ has been verified for dimension two and three (Hidano-Tsutaya [8] and Tzvetkov [41]), as well as radial cases in higher dimensions (Hidano-Wang-Yokoyama [10]). Notice however that the method which relies on Strichartz estimates yields small data global existence in $H^{s_{c}}$ for $p>\max ((n+$ $3) /(n-1), 3)$. For the sub-critical case $1<p \leq p_{c}(n)$, when $(a, b)=(1,0)$, it is known from Zhou [44] that there are no global solutions in general and an upper bound of the lifespan is given by

$$
T_{\varepsilon} \leq \begin{cases}\exp \left(C \varepsilon^{-(p-1)}\right), & p=p_{c}, \\ C \varepsilon^{\frac{2(p-1)}{(n-1)(p-1)-2}}, & 1<p<p_{c},\end{cases}
$$

for generic, compactly supported, smooth data of size $\varepsilon$.

${ }^{1}$ In Fang-Wang [3], the theorems were stated for equations of the form $\square u=\left|u_{t}\right|^{p-1} u_{t}$, however, as is clear from the proof, it also applies to (1.1) with $a>0$ and $b \geq 0$. 
Concerning long time well-posedness with small $H_{\text {rad }}^{s}$ data, it is known from [10] that $s=2$ is sufficient for existence. Moreover, the smallness of the initial data was measured in certain "multiplicative form", which strongly suggests that, the minimal regularity for the problem should be given by

$$
s_{o} \equiv \max \left(\frac{3}{2}, s_{c}\right)=\max \left(\frac{3}{2}, \frac{n+2}{2}-\frac{1}{p-1}\right) \text {. }
$$

Notice that for $p>1, s_{c}>3 / 2$ is equivalent to $p>p_{c}$.

By making use of the similar approach which is taken for (1.1), we prove that the problem (1.6) is locally well-posed in $H_{\text {rad }}^{s}$ for any $s \in\left(s_{o}, 2\right)$, when $1<p<$ $1+2 /(n-2)$. Furthermore, when $p_{c}<p<1+2 /(n-2)$, for any $s \in\left(s_{c}, 2\right)$, it admits global solution for small data in $H_{\text {rad }}^{s}$, which gives an affirmative answer for the natural regularity problem raised in [10].

The following is our result concerning the problem (1.6).

Theorem 1.3. Let $n \geq 2$ and $p \in(1,1+2 /(n-2))$ (when $n=2$, it means $p \in(1, \infty))$. The problem (1.6) is unconditionally well-posed in $H_{\mathrm{rad}}^{s}$ with $s \in$ $\left(s_{o}, 2\right)$, where $s_{o}$ is given by (1.7). More precisely, for any radial data $\left(u_{0}, u_{1}\right) \in$ $H_{\mathrm{rad}}^{s} \times H_{\mathrm{rad}}^{s-1}$, there exists $T \in(0, \infty]$, which depends only on the norm of $\left(u_{0}, u_{1}\right)$, such that the problem (1.6) admits a unique, radially symmetric solution $u \in$ $L^{\infty}\left([0, T) ; H_{\mathrm{rad}}^{s}\right) \cap \operatorname{Lip}\left([0, T) ; H_{\mathrm{rad}}^{s-1}\right)$. Moreover, $u \in C\left([0, T) ; H^{s}\right) \cap C^{1}\left([0, T) ; H^{s-1}\right)$,

$$
r^{-\frac{1}{2}+\delta}\langle r\rangle^{-\delta-\delta_{1}} \partial u \in L^{2}\left([0, T) \times \mathbb{R}^{n}\right),
$$

where $\delta \in(0,1 / 2)$ and $\delta_{1} \in[-\delta,(n-1) / 2)$ are defined by

$$
\delta=\left\{\begin{array}{ll}
\frac{p-1}{2}\left(s-s_{c}\right), & p \geq p_{c}, \\
\frac{p-1}{2}\left(\frac{3}{2}-s_{c}\right), & p<p_{c},
\end{array} \quad \delta_{1}= \begin{cases}\frac{n-1}{4} \min \left(1, p-p_{c}\right), & p>p_{c} \\
0, & p=p_{c} \\
-\delta, & p<p_{c} .\end{cases}\right.
$$

and there exist $c, \varepsilon_{0}>0$, such that we can choose $T=T_{\varepsilon}$, where

$$
T_{\varepsilon}= \begin{cases}c \varepsilon^{-\frac{1}{s-s_{c}}}, & \varepsilon \geq \varepsilon_{0}, p \geq p_{c}, \\ \exp \left(c \varepsilon^{-(p-1)}\right), & \varepsilon<\varepsilon_{0}, p=p_{c}, \\ \infty, & \varepsilon<\varepsilon_{0}, p>p_{c}, \\ c \varepsilon^{-\frac{1}{3 / 2-s_{c}}}=c \varepsilon^{\frac{2(p-1)}{(n-1)(p-1)-2}}, & p<p_{c},\end{cases}
$$

with

$$
\varepsilon= \begin{cases}\|\partial u(0)\|_{H^{s-1}}, & p>p_{c}, \\ \|\partial u(0)\|_{\dot{H}^{s-1}}+\|\partial u(0)\|_{\dot{H}^{s-1}}^{1 / 2}\|\partial u(0)\|_{\dot{H}^{2-s}}^{1 / 2}, & p=p_{c} \\ \|\partial u(0)\|_{\dot{H}^{s-1}}^{1 / 2}\|\partial u(0)\|_{\dot{H}^{2-s}}^{1 / 2}, & p<p_{c}\end{cases}
$$

Remark 1.4. For the two dimensional critical case $p=p_{c}(2)=3$, Fang-Wang [6] proved the same result for general data in $H^{s}$ with $s>s_{c}$, by assuming additional angular regularity $^{2}$ of order bigger than $1 / 2$.

As we have pointed out in Remark 1.2, Theorem 1.3 for $p \geq p_{c}$ fails to be true for $s=s_{c}$, in the sense that there exists a family of data such that the lifespans tend to zero while the data remain bounded in $H_{\mathrm{rad}}^{s_{c}}$. Nevertheless, it does not exclude the possibility of small data global existence in the critical space $H_{\mathrm{rad}}^{s_{c}}$, for $p>p_{c}$. Thus, one may naturally ask whether it is possible to obtain small data

\footnotetext{
${ }^{2}$ As for the meaning of additional angular regularity, see Lemma 2.1 below. There, we use the Sobolev space on the unit sphere
} 
global existence for $s=s_{c}$ under the assumption $p>p_{c}$. It turns out that this is true when the spatial dimension is two, while the higher dimensional cases remain open.

By exploiting generalized Strichartz estimates of Fang-Wang [4], Smith-SoggeWang [34], we obtain the following:

Theorem 1.4. Let $n=2$ and $p>5$. Then there exists a small constant $\varepsilon_{0}>0$, such that the Cauchy problem (1.6) has a unique global solution satisfying $u \in$ $C\left([0, \infty) ; H^{s_{c}}\right) \cap C^{1}\left([0, \infty) ; H^{s_{c}-1}\right)$ and $\partial u \in L_{t}^{p-1} L_{x}^{\infty}$, whenever the initial data $\left(u_{0}, u_{1}\right) \in H^{s_{c}} \times H^{s_{c}-1}$ with

$$
\left\|\left(u_{0}, u_{1}\right)\right\|_{\dot{H}^{s_{c}} \times \dot{H}^{s_{c}-1}}=\varepsilon \leq \varepsilon_{0} .
$$

In addition, there exists a consant $C>0$, such that the solution satisfies

$$
\|\partial u\|_{L_{t}^{\infty} \dot{H}^{s_{c}-1} \cap L_{t}^{p-1} L_{x}^{\infty}} \leq C \varepsilon,\|\partial u\|_{L_{t}^{\infty} L_{x}^{2}} \leq C\left\|\left(u_{0}, u_{1}\right)\right\|_{\dot{H}^{1} \times L^{2}} .
$$

Moreover, when $p>3$ and the initial data are radial, the same result remains valid for the radial solutions.

As we have recalled, $s_{c}$ and $s_{l}$ are believed to be lower bounds for the problem to be locally well-posed in $H^{s}$, which has been partially verified in Lindblad [23], [24], Fang-Wang [3], for $n \geq 3$. The necessity of $s \geq \max \left(s_{c}, s_{l}\right)$ for $n=2$ is more delicate, but nevertheless is still true, see Liu-Wang [26]. Since $s_{l}>s_{c}$ for $p<5$, we see that the condition $p \geq 5$ is necessary in general for (1.6) to be well-posed in $H^{s_{c}} \times H^{s_{c}-1}$.

Concerning the paper. Our paper is organized as follows. In the next section, we collect various basic estimates which we need, including trace estimates, Morawetz type estimates and Strichartz type estimates. In particular, we prove a weighted fractional chain rule, Theorem 1.2. Then in Section 3, we prove local well-posedness for (1.1) and (1.6), Theorem 1.1 and Theorem 1.3. In the last section, we prove Theorem 1.4, the small data global existence with critical regularity for dimension two.

\section{Preliminaries}

In this section, we collect various basic estimates to be used for proofs of theorems. All of these estimates are well known, except a weighted fractional chain rule, Theorem 1.2.

2.1. Trace estimates: spatial decay. At first, let us record the trace estimates, which will provide spatial decay for functions, see, e.g., (1.3), (1.7) in Fang-Wang $[5]$.

Lemma 2.1 (Trace estimates). Let $n \geq 2$ and $1 / 2<s<n / 2$. Then we have

$$
\left\|r^{n / 2-s} f\right\|_{L_{r}^{\infty} H_{\omega}^{s-1 / 2}} \lesssim\|f\|_{\dot{H}^{s}},\left\|r^{(n-1) / 2} f\right\|_{L_{r}^{\infty} L_{\omega}^{2}} \lesssim\|f\|_{\dot{B}_{2,1}^{1 / 2}}
$$

for any $f \in C_{0}^{\infty}\left(\mathbb{R}^{n}\right)$. Here $\dot{B}_{p, q}^{s}$ is the homogeneous Besov space (see, e.g., [4] for the definition) and $H_{\omega}^{s}=\left(1-\Delta_{\omega}\right)^{-s / 2} L_{\omega}^{2}$ is the Sobolev space on the unit sphere. In particular, when $u$ is spatially radial, then $|\partial u| \lesssim\|\partial u(t, r \cdot)\|_{L_{\omega}^{2}}$ and

$$
\left\|r^{n / 2-s} \partial u\right\|_{L_{t, x}^{\infty}} \lesssim\|\partial u\|_{L_{t}^{\infty} \dot{H}^{s}},\left\|r^{(n-1) / 2} \partial u\right\|_{L_{t, x}^{\infty}}^{2} \lesssim\|\partial u\|_{L_{t}^{\infty} \dot{H}^{s}}\|\partial u\|_{L_{t}^{\infty} \dot{H}^{1-s}} .
$$


2.2. Space-time estimates. We will need to exploit the following space-time estimates for solutions to the linear wave equations: Morawetz type (local energy) estimates, as well as the generalized Strichartz estimates.

At first, we record the required Morawetz type estimates for the operator $\square$.

Lemma 2.2. Let $n \geq 2$. Then for any $\delta \in(0,1 / 2]$ and $\delta_{1}>0$, there exists $C>0$, such that we have

$$
\begin{aligned}
& T^{-\delta}\left\|r^{-\frac{1}{2}+\delta} \partial u\right\|_{L_{T}^{2} L_{x}^{2}}+\|\partial u\|_{L_{T}^{\infty} L_{x}^{2}} \leq C\|\partial u(0)\|_{L_{x}^{2}}+C T^{\delta}\left\|r^{\frac{1}{2}-\delta} \square u\right\|_{L_{T}^{2} L_{x}^{2}}, \\
& A_{T}^{-1}\left\|r^{-\frac{1}{2}+\delta}\langle r\rangle^{-\delta} \partial u\right\|_{L_{T}^{2} L_{x}^{2}}+\|\partial u\|_{L_{T}^{\infty} L_{x}^{2}} \\
& \leq C\|\partial u(0)\|_{L_{x}^{2}}+C A_{T}\left\|r^{\frac{1}{2}-\delta}\langle r\rangle^{\delta} \square u\right\|_{L_{T}^{2} L_{x}^{2}}, \\
& B_{T}^{-1}\left\|r^{-\frac{1}{2}+\delta}\langle r\rangle^{-\delta-\delta_{1}} \partial u\right\|_{L_{T}^{2} L_{x}^{2}}+\|\partial u\|_{L_{T}^{\infty} L_{x}^{2}} \\
& \leq C\|\partial u(0)\|_{L_{x}^{2}}+C B_{T}\left\|r^{\frac{1}{2}-\delta}\langle r\rangle^{\delta+\delta_{1}} \square u\right\|_{L_{T}^{2} L_{x}^{2}},
\end{aligned}
$$

for any $T \in(0, \infty)$, where $A_{T}=\min \left(T^{\delta}, \sqrt{\ln (2+T)}\right), B_{T}=\min \left(T^{\delta}, 1\right), L_{T}^{q}=$ $L^{q}([0, T))$ for the variable $t$ and $C$ is independent of $T>0$.

The above estimates were formulated and proved in [10, Lemma 3.2] for $n \geq 3$, by multiplier method, see also $[27,11,42]$. We remark that the estimates with $n \geq 2$ is also implied by the local energy estimates in Metcalfe-Tataru [28, Theorem 1], and a well-known argument of Keel-Smith-Sogge [18]. Morawetz type estimates and local energy estimates have rich history and a large body of related literature. We refer the interested readers to $[28,25]$ for more exhaustive treatment of such estimates.

For the problem with dimension two and critical regularity, we will also use the following generalized Strichartz estimates due to Smith-Sogge-Wang [34], along with the previous radial estimates in Fang-Wang [4]. See also [14] for similar results in higher dimensions. For Strichartz estimates, see [4] and references therein.

Lemma 2.3 (Generalized Strichartz estimates). Let $n=2, q \in(2,4]$, and $s=$ $1-1 / q$. Then we have the following inequality

$$
\|\partial u\|_{L^{q}\left([0, \infty) ; L_{r}^{\infty} L_{\omega}^{2}\left(\mathbb{R}^{2}\right)\right)} \lesssim\|\partial u(0)\|_{\dot{H}_{x}^{s}}+\|\square u\|_{L_{t}^{1} \dot{H}_{x}^{s}} .
$$

In addition, we have the classical Strichartz estimates for $q \in(4, \infty)$,

$$
\|\partial u\|_{L^{q}\left([0, \infty) ; L_{x}^{\infty}\left(\mathbb{R}^{2}\right)\right)} \lesssim\|\partial u(0)\|_{\dot{H}_{x}^{s}}+\|\square u\|_{L_{t}^{1} \dot{H}_{x}^{s}} .
$$

In particular, when $u$ is (spatially) radial, we have

$$
\|\partial u\|_{L^{q}\left([0, \infty) ; L_{x}^{\infty}\left(\mathbb{R}^{2}\right)\right)} \lesssim\|\partial u(0)\|_{\dot{H}_{x}^{s}}+\|\square u\|_{L_{t}^{1} \dot{H}_{x}^{s}}, q>2 .
$$

As mentioned in Lemma 2.1, we have $|\partial u(t, x)| \leq C\|\partial u(t, r \cdot)\|_{L_{\omega}^{2}}$, when $u$ is spatially radial. Combining (2.6) with it, we easily obtain (2.8), which means that the 2-D radial estimate due to Fang-Wang (see Theorem 4 in [4]) remains true for the first derivatives of radial solutions. 
2.3. Weighted fractional chain rule. To apply the Morawetz type estimates for the nonlinear problem, we are naturally required to introduce the weighted fractional chain rule, Theorem 1.2. Before proving it in Section 2.4, we firstly discuss the implication which will be useful for our problem.

As a direct corollary of Theorem 1.2, with $w_{2}=w_{1}^{-2}=w^{-1}$ and the fact that

$$
w \in A_{1} \Rightarrow w \in A_{2} \Rightarrow w^{-1} \in A_{2},
$$

we obtain the following:

Corollary 2.4. Let $w \in A_{1}$ and $s \in(0,1)$. Under the same assumption on $F(u)$ as in Theorem 1.2, we have

$$
\left\|w^{-1 / 2} D^{s} F(u)\right\|_{L_{x}^{2}} \lesssim\left\|w^{1 / 2} D^{s} u\right\|_{L_{x}^{2}}\left\|w^{-1} G(u)\right\|_{L_{x}^{\infty}} .
$$

The actual weight function we will choose is of the form $w(x)=r^{-1+2 \delta}\langle r\rangle^{-2 \delta-2 \delta_{1}}$.

Lemma 2.5. Let $w(x)=r^{-1+2 \delta}\langle r\rangle^{-2 \delta-2 \delta_{1}}$, with $0 \leq 1-2 \delta \leq 1+2 \delta_{1}<n$. Then $w \in A_{1}\left(\mathbb{R}^{n}\right)$.

Proof. Though proof of this lemma is rather elementary, for the sake of completeness we provide a proof. It amounts to proving that for any $r>0$, and almost every $x \in \mathbb{R}^{n}$,

$$
\int_{B_{r}(x)}|y|^{-1+2 \delta}\langle y\rangle^{-2 \delta-2 \delta_{1}} d y \leq C r^{n}|x|^{-1+2 \delta}\langle x\rangle^{-2 \delta-2 \delta_{1}} .
$$

We deal with two cases separately. First, if $|x| \leq 1$, then as $\delta+\delta_{1} \geq 0$, we have

$$
|y|^{-1+2 \delta}\langle y\rangle^{-2 \delta-2 \delta_{1}} \leq|y|^{-1+2 \delta} \in A_{1}
$$

provided that $1-2 \delta \in[0, n)$ (recall that $|x|^{a} \in A_{1}$ iff $a \in(-n, 0]$, Grafakos [7, Example 7.1.7, page 506]). Thus,

$$
r^{-n} \int_{B_{r}(x)}|y|^{-1+2 \delta}\langle y\rangle^{-2 \delta-2 \delta_{1}} d y \leq C|x|^{-1+2 \delta} \leq C|x|^{-1+2 \delta}\langle x\rangle^{-2 \delta-2 \delta_{1}}, \forall r>0 .
$$

For the case $|x| \geq 1$, recall that $|x|^{-1-2 \delta_{1}} \in A_{1}$ if $1+2 \delta_{1} \in[0, n)$. So, we have

$$
r^{-n} \int_{B_{r}(x)}|y|^{-1-2 \delta_{1}} d y \leq C|x|^{-1-2 \delta_{1}}, \forall r>0 .
$$

If $r<|x| / 2$, we have $|y| \geq|x|-r \geq|x| / 2$ and so $|y| \simeq\langle y\rangle \simeq|x|$ for $y \in B_{r}(x)$. Hence,

$$
r^{-n} \int_{B_{r}(x)}|y|^{-1+2 \delta}\langle y\rangle^{-2 \delta-2 \delta_{1}} d y \leq C r^{-n} \int_{B_{r}(x)}|x|^{-1-2 \delta_{1}} d y \leq C|x|^{-1+2 \delta}\langle x\rangle^{-2 \delta-2 \delta_{1}} .
$$

On the other hand, if $r \geq|x| / 2$, by (2.11), it follows that

$$
\begin{aligned}
\int_{B_{r}(x)}|y|^{-1+2 \delta}\langle y\rangle^{-2 \delta-2 \delta_{1}} d y & \leq \int_{B_{1}}|y|^{-1+2 \delta} d y+\int_{B_{r}(x) \backslash B_{1}}|y|^{-1-2 \delta_{1}} d y \\
& \leq C+C r^{n}|x|^{-1-2 \delta_{1}} \\
& \leq C r^{n}|x|^{-1-2 \delta_{1}} .
\end{aligned}
$$

This gives the desired (2.10) and completes the proof. 
2.4. Proof of Theorem 1.2. To begin with, we recall that if $T$ is a strong Calderón-Zygmund operator, then

$$
\|T(f)\|_{L^{p}(w d x)} \leq C\|f\|_{L^{p}(w d x)}, w \in A_{p}, p \in(1, \infty) .
$$

(See, e.g., Muscalu-Schlag [29, Theorem 7.21, page 191].) Using this and the argument in $[29$, Section 8.2] it is easy to see that the weighted Littlewood-Paley square-function estimate

$$
\left\|w S_{j} f\right\|_{L^{p} \ell_{j}^{2}} \simeq\|w f\|_{L^{p}}, w^{p} \in A_{p}, f \in L^{p}\left(w^{p} d x\right), p \in(1, \infty)
$$

holds where $S_{j}=\phi_{j} *$ is the standard Littlewood-Paley operator while $\phi_{j}(x)=$ $2^{j n} \phi\left(2^{j} x\right)$, supp $\hat{\phi} \subset\left\{|\xi| \in\left[2^{-2}, 2^{2}\right]\right\}$.

By repeating essentially the same argument as in Taylor [40, (5.6), page 112], we can obtain

$$
\left|S_{j} D^{s} F(u)(x)\right| \lesssim 2^{j s} \sum_{k \in \mathbb{Z}} \min \left(1,2^{k-j}\right)\left(\mathcal{M}\left(S_{k} u\right)(x) \mathcal{M} H(x)+\mathcal{M}\left(H S_{k} u\right)(x)\right),
$$

where $H(x) \equiv G(u(x))$. By $(2.13)$ and $(2.14)$, we know that for $\left(w_{1}\right)^{q},\left(w_{1} w_{2}\right)^{q} \in A_{q}$ with $q \in(1, \infty)$,

$$
\begin{aligned}
& \left\|w_{1} w_{2} D^{s} F(u)\right\|_{L^{q}} \lesssim\left\|w_{1} w_{2} S_{j} D^{s} F(u)\right\|_{L^{q} \ell_{j}^{2}} \\
\lesssim & \left\|w_{1} w_{2} 2^{j s} \min \left(1,2^{k-j}\right)\left(\mathcal{M}\left(S_{k} u\right) \mathcal{M} H+\mathcal{M}\left(H S_{k} u\right)\right)\right\|_{L^{q} \ell_{j}^{2} \ell_{k}^{1}} \\
\lesssim & \left\|w_{1} w_{2} 2^{k s} \min \left(2^{(j-k) s}, 2^{(k-j)(1-s)}\right)\left(\mathcal{M}\left(S_{k} u\right) \mathcal{M} H+\mathcal{M}\left(H S_{k} u\right)\right)\right\|_{L^{q} \ell_{j}^{2} \ell_{k}^{1}} \\
\lesssim & \left\|w_{1} w_{2} 2^{k s}\left(\mathcal{M}\left(S_{k} u\right) \mathcal{M} H+\mathcal{M}\left(H S_{k} u\right)\right)\right\|_{L^{q} \ell_{k}^{2}} .
\end{aligned}
$$

For the last inequality we use Young's inequality with the assumption $s \in(0,1)$.

By applying Minkowski's and Hölder's inequalities to the last expression we have

$$
\begin{gathered}
\left.\left\|w_{1} w_{2} D^{s}(F(u))\right\|_{L^{q}} \lesssim\left\|w_{1} w_{2} 2^{k s} \mathcal{M}\left(S_{k} u\right) \mathcal{M} H\right\|_{L^{q} \ell_{k}^{2}}+\| w_{1} w_{2} 2^{k s} \mathcal{M}\left(H S_{k} u\right)\right) \|_{L^{q} \ell_{k}^{2}} \\
\lesssim\left\|w_{2} \mathcal{M} H\right\|_{L^{q_{2}}}\left\|w_{1} 2^{k s} \mathcal{M}\left(S_{k} u\right)\right\|_{L^{q_{1} \ell_{k}^{2}}}+\left\|w_{1} w_{2} 2^{k s} H S_{k} u\right\|_{L^{q} \ell_{k}^{2}},
\end{gathered}
$$

for any $q_{1}, q_{2} \in(1, \infty]$ with $\frac{1}{q}=\frac{1}{q_{1}}+\frac{1}{q_{2}}$. For the last term in the above we used the weighted vector valued inequality for the Hardy-Littlewood inequality which is due to Andersen-John [1, Theorem 3.1]:

$$
\int\left\|\mathcal{M} f_{j}\right\|_{\ell_{j}^{p}}^{q} w d x \leq C \int\left\|f_{j}\right\|_{\ell_{j}^{p}}^{q} w d x, p, q \in(1, \infty), w \in A_{q} .
$$

Using (2.15) again and Hölder's inequality,

$\left\|w_{1} w_{2} D^{s}(F(u))\right\|_{L^{q}} \lesssim\left\|w_{2} \mathcal{M} H\right\|_{L^{q_{2}}}\left\|w_{1} 2^{k s} S_{k} u\right\|_{L^{q_{1}} \ell_{k}^{2}}+\left\|w_{2} H\right\|_{L^{q_{2}}}\left\|w_{1} 2^{k s} S_{k} u\right\|_{L^{q_{1}} \ell_{k}^{2}}$.

Then, by (2.13) and its variant

$$
\left\|w_{1} 2^{k s} S_{k} u\right\|_{L^{q_{1}} \ell_{k}^{2}} \equiv\left\|w_{1} \tilde{S}_{k} D^{s} u\right\|_{L^{q_{1} \ell_{k}^{2}}} \lesssim\left\|w_{1} D^{s} u\right\|_{L^{q_{1}}}, \quad w_{1} \in A_{q_{1}}, q_{1} \in(1, \infty),
$$

we get that

$$
\left\|w_{1} w_{2} D^{s}(F(u))\right\|_{L^{q}} \lesssim\left(\left\|w_{2} \mathcal{M} H\right\|_{L^{q_{2}}}+\left\|w_{2} H\right\|_{L^{q_{2}}}\right)\left\|w_{1} D^{s} u\right\|_{L^{q_{1}}} .
$$

If $q_{2}<\infty$, (1.4) follows directly from (2.16), by applying (2.15) for the term involving $\mathcal{M H}$. To handle the remaining case $q_{2}=\infty$, we observe that for $w \in A_{1}$,

$$
\left\|w^{-1} \mathcal{M} H\right\|_{L^{\infty}} \lesssim\left\|w^{-1} H\right\|_{L^{\infty}} .
$$


This is trivial, since by the definition of $A_{1}$, we know that for a.e. $x \in \mathbb{R}^{n}$,

$$
\begin{gathered}
H(x) \leq w(x)\left\|w^{-1} H\right\|_{L^{\infty}} \Rightarrow \\
\mathcal{M} H(x) \leq \mathcal{M} w(x)\left\|w^{-1} H\right\|_{L^{\infty}} \leq C w(x)\left\|w^{-1} H\right\|_{L^{\infty}},
\end{gathered}
$$

which gives (2.17). Together with (2.16), we get (1.5) and this completes the proof.

\section{LOCAL WELL-POSEDNESS}

In this section, we prove local well-posedness for (1.1) and (1.6), Theorem 1.1 and Theorem 1.3, based on Lemmas 2.1-2.2 and Theorem 1.2. As (1.1) is a special case of (1.6), with $n=3$ and $p=2$, we need only to prove Theorem 1.3.

3.1. Local existence. As usual, we prove the existence of solutions for (1.6) through iteration. For fixed $s \in\left(s_{o}, 2\right)$ and $\left(u_{0}, u_{1}\right) \in H_{\text {rad }}^{s} \times H_{\text {rad }}^{s-1}$, we define the iteration map

$$
\Phi[u]:=H\left[u_{0}, u_{1}\right]+I[N[u]],
$$

where $H[\phi, \psi]=\cos (t D) \phi+D^{-1} \sin (t D) \psi$ is the solution map of the linear homogeneous Cauchy problem with data $(\phi, \psi), I[F](t, \cdot)=\int_{0}^{t} D^{-1} \sin ((t-\tau) D) F(\tau, \cdot) d \tau$ is the solution map of the linear inhomogeneous Cauchy problem $\square u=F$ with vanishing data, and the nonlinear term

$$
N[u]:=a\left|\partial_{t} u\right|^{p}+b\left|\nabla_{x} u\right|^{p} .
$$

Notice that $\Phi$ preserves radial property and we have

$$
D^{\nu} \Phi[u]=H\left[D^{\nu} u_{0}, D^{\nu} u_{1}\right]+I\left[D^{\nu} N[u]\right],
$$

for any $\nu \in(0,1)$.

Let $w(x)=r^{-1+2 \delta}\langle r\rangle^{-2 \delta-2 \delta_{1}}$ with $\delta \in(0,1 / 2)$ and $\delta_{1} \in[-\delta,(n-1) / 2)$ be defined by (1.8). Applying Lemma 2.2 for $D^{\nu} \Phi[u]$ with $\nu \in[0,1)$, we get, for $T>0$ to be determined later,

$$
\|\Phi[u]\|_{X_{\nu}^{T}} \lesssim\|\partial u(0)\|_{\dot{H}^{\nu}}+\tilde{A}_{T}\left\|w^{-\frac{1}{2}} D^{\nu} N[u]\right\|_{L_{T}^{2} L_{x}^{2}},
$$

where

$$
\begin{gathered}
\|u\|_{X_{\nu}^{T}}=\tilde{A}_{T}^{-1}\left\|w^{\frac{1}{2}} \partial D^{\nu} u\right\|_{L_{T}^{2} L_{x}^{2}}+\|\partial u\|_{L_{T}^{\infty} \dot{H}_{x}^{\nu}}, \\
\tilde{A}_{T}= \begin{cases}B_{T}=\min \left(T^{\delta}, 1\right), & p>p_{c}, \\
A_{T}=\min \left(T^{\delta}, \sqrt{\ln (2+T)}\right), & p=p_{c}, \\
T^{\delta}, & p<p_{c}\end{cases}
\end{gathered}
$$

As $\delta \in(0,1 / 2]$ and $\delta_{1} \in[-\delta,(n-1) / 2)$, we can apply Lemma 2.5 and Corollary 2.4 to conclude

$$
\|\Phi[u]\|_{X_{\nu}^{T}} \lesssim\|\partial u(0)\|_{H^{\nu}}+\tilde{A}_{T}^{2}\|u\|_{X_{\nu}^{T}}\left\|w^{-1}|\partial u|^{p-1}\right\|_{L_{T}^{\infty} L_{x}^{\infty}}, \nu \in(0,1) .
$$

The case $\nu=0$ is also admissible, by Hölder's inequality.

We now note that the choice of $\delta, \delta_{1}$ ensures that

$$
\frac{1-2 \delta}{p-1}= \begin{cases}\frac{n}{2}-(s-1), & p \geq p_{c} \\ \frac{n-1}{2}, & p<p_{c}\end{cases}
$$


$\frac{1+2 \delta_{1}}{p-1} \in\left(\frac{n}{2}-(s-1), \frac{n-1}{2}\right]$ when $p>p_{c}$ and $\frac{1+2 \delta_{1}}{p-1}=\frac{n-1}{2}$ when $p \leq p_{c}$. Thus by (2.2), we see that for radial $u$,

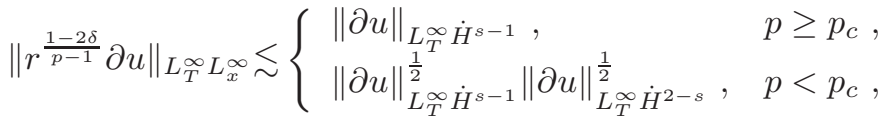

$$
\begin{aligned}
& \left\|r^{\frac{1+2 \delta_{1}}{p-1}} \partial u\right\|_{L_{T}^{\infty} L_{x}^{\infty}} \lesssim \begin{cases}\|\partial u\|_{L_{T}^{\infty} H^{s-1}}, & p>p_{c} \\
\|\partial u\|_{L_{T}^{\infty} \dot{H}^{s-1}}^{\frac{1}{2}}\|\partial u\|_{L_{T}^{\infty} \dot{H}^{2-s}}^{\frac{1}{2}}, & p \leq p_{c} .\end{cases}
\end{aligned}
$$

In conclusion, we arrive at, for any $\nu \in[0,1)$,

$$
\begin{array}{r}
\|\Phi[u]\|_{X_{\nu}^{T}} \leq C_{\nu}\|\partial u(0)\|_{\dot{H}^{\nu}} \\
+C_{\nu} \tilde{A}_{T}^{2}\|u\|_{X_{\nu}^{T}} \times \begin{cases}\left(\|u\|_{X_{s-1}^{T}}+\|u\|_{X_{0}^{T}}\right)^{p-1}, & p>p_{c}, \\
\left(\|u\|_{X_{s-1}^{T}}^{2}+\|u\|_{X_{s-1}^{T}}\|u\|_{X_{2-s}^{T}}\right)^{\frac{p-1}{2}}, & p=p_{c}, \\
\left(\|u\|_{X_{s-1}^{T}}\|u\|_{X_{2-s}^{T}}\right)^{\frac{p-1}{2}}, & p<p_{c} .\end{cases}
\end{array}
$$

Similarly, as $|N[u]-N[v]| \lesssim\left(|\partial u|^{p-1}+|\partial v|^{p-1}\right)|\partial(u-v)|$, we have

$$
\begin{aligned}
\| \Phi[u] & -\Phi[v]\left\|_{X_{0}^{T}} \lesssim \tilde{A}_{T}^{2}\right\| u-v \|_{X_{0}^{T}} \\
& \times \begin{cases}\left(\|(u, v)\|_{X_{s-1}^{T}}+\|(u, v)\|_{X_{0}^{T}}\right)^{p-1}, & p>p_{c}, \\
\left(\|(u, v)\|_{X_{s-1}^{T}}^{2}+\|(u, v)\|_{X_{s-1}^{T}}\|(u, v)\|_{X_{2-s}^{T}}\right)^{\frac{p-1}{2}}, & p=p_{c}, \\
\left(\|(u, v)\|_{X_{s-1}^{T}}\|(u, v)\|_{X_{2-s}^{T}}\right)^{\frac{p-1}{2}}, & p<p_{c} .\end{cases}
\end{aligned}
$$

Based on (3.5) with $\nu=s-1,2-s, 0$ and (3.6), it is standard to conclude that there exist $c, \varepsilon_{0}$ such that for $T_{\varepsilon}$ given in (1.9), $\Phi$ is a contraction mapping in the complete metric space

$$
\begin{aligned}
\left\{u \in L^{\infty}\left([0, T) ; H_{\mathrm{rad}}^{s}\right)\right. & \cap \operatorname{Lip}\left([0, T) ; H_{\mathrm{rad}}^{s-1}\right), \\
& \left.\|\partial u\|_{L_{T}^{\infty} \dot{H}^{\nu}} \leq 2 C_{\nu}\|\partial u(0)\|_{\dot{H}^{\nu}}, \nu=s-1,2-s, 0\right\}
\end{aligned}
$$

with the metric $d(u, v)=\|u-v\|_{X_{0}^{T}}$, and the unique fixed point is the solution we are seeking. This completes the proof of the existence part of Theorem 1.3.

3.2. Unconditional uniqueness. Suppose we have two radial solutions $u, v \in$ $L^{\infty}\left([0, T) ; H_{\mathrm{rad}}^{s}\right) \cap \operatorname{Lip}\left([0, T) ; H_{\mathrm{rad}}^{s-1}\right)$ to $(1.6)$.

Observing that $s>s_{o} \geq 3 / 2$, we have $s-1>1 / 2>1 / 2-\delta$, and so

$$
\left\|w^{\frac{1}{2}} \partial u(t)\right\|_{L^{2}} \lesssim\left\|r^{-1 / 2+\delta} \partial u(t)\right\|_{L^{2}} \lesssim\|\partial u(t)\|_{H^{1 / 2-\delta}} \lesssim\|\partial u(t)\|_{H^{s-1}},
$$

where we have used Hardy's inequality in the second inequality. Thus, $w^{\frac{1}{2}} \partial(u, v) \in$ $L_{\text {loc }}^{2}\left([0, T) ; L^{2}\right)$. Then, as in the proof of the existence, we get from Lemma 2.2, (3.3) and (3.4) that, for any $T_{1} \in(0, T)$ and $T_{1}<1$,

$$
\begin{gathered}
\left\|w^{\frac{1}{2}} \partial(u-v)\right\|_{L_{T_{1}}^{2} L_{x}^{2}} \lesssim T_{1}{ }^{2 \delta}\left\|w^{-\frac{1}{2}} \square(u-v)\right\|_{L_{T_{1}}^{2} L_{x}^{2}} \\
\quad \lesssim T_{1}{ }^{2 \delta}\|\partial(u, v)\|_{L_{T}^{\infty} H^{s-1}}^{p-1}\left\|w^{\frac{1}{2}} \partial(u-v)\right\|_{L_{T_{1}}^{2} L_{x}^{2}} .
\end{gathered}
$$

Letting $T_{1}$ sufficiently small such that $T_{1}^{2 \delta}\|\partial(u, v)\|_{L_{T}^{\infty} H^{s-1}}^{p-1} \ll 1$, we conclude that $u \equiv v$ in $\left[0, T_{1}\right] \times \mathbb{R}^{n}$, which yields unconditional uniqueness. 
3.3. Regularity. In this subsection, we prove the improved regularity of the solution $u \in C\left([0, T) ; H^{s}\right) \cap C^{1}\left([0, T) ; H^{s-1}\right)$, for which we need only to prove the continuity at $t=0$ of $\partial_{t}^{j} u(t) \in \dot{H}^{s-j}\left(\mathbb{R}^{n}\right), j=0,1$. Applying Corollary 2.4, (3.3) and (3.4), and recalling the fact that $w^{\frac{1}{2}} \partial D^{s-1} u \in L_{T}^{2} L_{x}^{2}$, we have for any $T_{1} \in(0, T)$ and $T_{1}<1$,

$$
\begin{aligned}
\left\|\partial u\left(T_{1}\right)-\partial u(0)\right\|_{\dot{H}_{x}^{s-1}} & =\left\|\partial \Phi[u]\left(T_{1}\right)-\partial u(0)\right\|_{\dot{H}_{x}^{s-1}} \\
& \left.\leq\left\|\partial I[N[u]]\left(T_{1}\right)\right\|_{\dot{H}_{x}^{s-1}}+\| \partial H\left[u_{0}, u_{1}\right]\left(T_{1}\right)-\partial u(0)\right) \|_{\dot{H}_{x}^{s-1}} \\
& \lesssim T_{1}^{\delta}\left\|w^{-\frac{1}{2}} D^{s-1} N[u]\right\|_{L_{T_{1}}^{2} L_{x}^{2}}+o(1) \\
& \lesssim T_{1}^{\delta}\|\partial u\|_{L_{T_{1}}^{\infty} H^{s-1}}^{p-1}\left\|w^{\frac{1}{2}} \partial D^{s-1} u\right\|_{L_{T_{1}}^{2} L_{x}^{2}}+o(1)=o(1)
\end{aligned}
$$

as $T_{1} \rightarrow 0+$, which proves the continuity at $t=0$.

\section{Small data Global existence With CRitical Regularity}

In this section, focusing on spatial dimension two, we use the generalized Strichartz estimates to prove Theorem 1.4, which yields the small data global existence with critical regularity.

Firstly, we note that $s_{c}=2-1 /(p-1) \in(1,2)$ when $p>5$. Applying (2.7) of Lemma 2.3 and energy estimates we get

$$
\begin{aligned}
& \left\|\left(\Phi[u], \partial_{t} \Phi[u]\right)\right\|_{L_{t}^{\infty}\left(\dot{H}^{s_{c}} \times \dot{H}^{s_{c}-1}\right)}+\|\partial \Phi[u]\|_{L_{t}^{p-1} L_{x}^{\infty}} \\
\lesssim & \left\|\left(u_{0}, u_{1}\right)\right\|_{\dot{H}^{s_{c}} \times \dot{H}^{s_{c}-1}}+\|N[u]\|_{L_{t}^{1} \dot{H}^{s_{c}-1}} \\
\lesssim & \varepsilon+\|\partial u\|_{L_{t}^{p-1} L_{x}^{\infty}}^{p-1}\|\partial u\|_{L_{t}^{\infty} \dot{H}^{s_{c}-1}} .
\end{aligned}
$$

In the last inequality, we use Theorem 1.2 with $w_{1}=w_{2}=1, F(v)=|v|^{p}, G(v)=$ $F^{\prime}(v)$ and $v=\partial u$. Similarly, by energy estimates, we have

$$
\begin{aligned}
\left\|\left(\Phi[u], \partial_{t} \Phi[u]\right)\right\|_{L_{t}^{\infty}\left(\dot{H}^{1} \times L^{2}\right)} & \lesssim\left\|\left(u_{0}, u_{1}\right)\right\|_{\dot{H}^{1} \times L^{2}}+\|N[u]\|_{L_{t}^{1} L_{x}^{2}} \\
& \lesssim\left\|\left(u_{0}, u_{1}\right)\right\|_{\dot{H}^{1} \times L^{2}}+\|\partial u\|_{L_{t}^{p-1} L_{x}^{\infty}}^{p-1}\|\partial u\|_{L_{t}^{\infty} L_{x}^{2}} .
\end{aligned}
$$

Moreover, as $\Phi[u]$ and $\Phi[v]$ have the same data, we have

$$
\begin{aligned}
& \left\|\left(\Phi[u]-\Phi[v], \partial_{t}(\Phi[u]-\Phi[v])\right)\right\|_{L_{t}^{\infty}\left(\dot{H}^{1} \times L^{2}\right)} \\
\lesssim & \|N[u]-N[v]\|_{L_{t}^{1} L_{x}^{2}} \\
\lesssim & \left(\|\partial u\|_{L_{t}^{p-1} L_{x}^{\infty}}^{p-1}+\|\partial v\|_{L_{t}^{p-1} L_{x}^{\infty}}^{p-1}\right)\|\partial(u-v)\|_{L_{t}^{\infty} L_{x}^{2}} .
\end{aligned}
$$

Once we have the above three estimates, it is easy and rather standard to prove the existence and uniqueness for (1.6) in $C_{t} H^{s_{c}} \cap C_{t}^{1} H^{s_{c}-1}$ with $\partial u \in L_{t}^{p-1} L_{x}^{\infty}$, when $(\phi, \psi) \in H^{s_{c}} \times H^{s_{c}-1}$ and $\varepsilon$ is small enough.

When $p>3$ and the initial data are radial, we use (2.8) of Lemma 2.3 instead of (2.7). Then the same proof as before applies without modification. This completes the proof of Theorem 1.4.

Acknowledgment. K. Hidano was supported in part by the Grant-in-Aid for Scientific Research (C) (No. 15K04955), Japan Society for the Promotion of Science (JSPS). J.-C. Jiang was supported by National Sci-Tech Grant MOST 104-2115M-007-002. S. Lee was supported by the grant No. NRF-2015R1A4A1041675. C. Wang was supported in part by NSFC 11301478 and National Support Program for Young Top-Notch Talents. 


\section{REFERENCES}

[1] K. F. Andersen and R. T. John. Weighted inequalities for vector-valued maximal functions and singular integrals. Studia Math., 69(1):19-31, 1980/81.

[2] F. M. Christ and M. I. Weinstein. Dispersion of small amplitude solutions of the generalized Korteweg-de Vries equation. J. Funct. Anal., 100(1):87-109, 1991.

[3] D. Fang and C. Wang. Local well-posedness and ill-posedness on the equation of type $\square u=$ $u^{k}(\partial u)^{\alpha}$. Chinese Ann. Math. Ser. B, 26(3):361-378, 2005.

[4] D. Fang and C. Wang. Some remarks on Strichartz estimates for homogeneous wave equation. Nonlinear Anal., 65(3):697-706, 2006.

[5] D. Fang and C. Wang. Weighted Strichartz estimates with angular regularity and their applications. Forum Math., 23(1):181-205, 2011.

[6] D. Fang and C. Wang. Almost global existence for some semilinear wave equations with almost critical regularity. Comm. Partial Differential Equations, 38(9):1467-1491, 2013.

[7] L. Grafakos. Classical Fourier analysis, volume 249 of Graduate Texts in Mathematics. Springer, New York, third edition, 2014.

[8] K. Hidano and K. Tsutaya. Global existence and asymptotic behavior of solutions for nonlinear wave equations. Indiana Univ. Math. J., 44(4):1273-1305, 1995.

[9] K. Hidano and C. Wang Fractional derivatives of composite functions and the Cauchy problem for the nonlinear half wave equation. arXiv:1707.08319.

[10] K. Hidano, C. Wang, and K. Yokoyama. The Glassey conjecture with radially symmetric data. J. Math. Pures Appl. (9), 98(5):518-541, 2012.

[11] K. Hidano, C. Wang, and K. Yokoyama. On almost global existence and local well posedness for some 3-D quasi-linear wave equations. Adv. Differential Equations, 17(3-4):267-306, 2012.

[12] K. Hidano and K. Yokoyama. Space-time $L^{2}$-estimates and life span of the KlainermanMachedon radial solutions to some semi-linear wave equations. Differential Integral Equations, 19(9):961-980, 2006.

[13] K. Hidano and D. Zha, Space-time $L^{2}$ estimates, regularity and almost global existence for elastic waves. arXiv:1710.05180.

[14] J.-C. Jiang, C. Wang, and X. Yu. Generalized and weighted Strichartz estimates. Commun. Pure Appl. Anal., 11(5):1723-1752, 2012.

[15] F. John and S. Klainerman. Almost global existence to nonlinear wave equations in three space dimensions. Comm. Pure Appl. Math., 37(4):443-455, 1984.

[16] F. John. Blow-up for quasilinear wave equations in three space dimensions. Comm. Pure Appl. Math., 34(1):29-51, 1981.

[17] T. Kato. On nonlinear Schrödinger equations. II. $H^{s}$-solutions and unconditional wellposedness. J. Anal. Math., 67:281-306, 1995.

[18] M. Keel, H. F. Smith, and C. D. Sogge. Almost global existence for some semilinear wave equations. J. Anal. Math., 87:265-279, 2002. Dedicated to the memory of Thomas H. Wolff.

[19] S. Klainerman and M. Machedon. Space-time estimates for null forms and the local existence theorem. Comm. Pure Appl. Math., 46(9):1221-1268, 1993.

[20] S. Klainerman and M. Machedon. Smoothing estimates for null forms and applications. Duke Math. J., 81(1):99-133 (1996), 1995. A celebration of John F. Nash, Jr.

[21] S. Klainerman and M. Machedon. Estimates for null forms and the spaces $H_{s, \delta}$. Internat. Math. Res. Notices, (17):853-865, 1996.

[22] S. Klainerman and S. Selberg. Remark on the optimal regularity for equations of wave maps type. Comm. Partial Differential Equations, 22(5-6):901-918, 1997.

[23] H. Lindblad. A sharp counterexample to the local existence of low-regularity solutions to nonlinear wave equations. Duke Math. J., 72(2):503-539, 1993.

[24] H. Lindblad. Counterexamples to local existence for semi-linear wave equations. Amer. J. Math., 118(1):1-16, 1996.

[25] H. Lindblad, J. Metcalfe, C. D. Sogge, M. Tohaneanu, and C. Wang. The Strauss conjecture on Kerr black hole backgrounds. Math. Ann., 359(3-4):637-661, 2014.

[26] M. Liu and C. Wang, Concerning ill-posedness for semilinear wave equations. in preparation.

[27] J. Metcalfe and C. D. Sogge. Long-time existence of quasilinear wave equations exterior to star-shaped obstacles via energy methods. SIAM J. Math. Anal., 38(1):188-209, 2006.

[28] J. Metcalfe and D. Tataru. Global parametrices and dispersive estimates for variable coefficient wave equations. Math. Ann., 353(4):1183-1237, 2012. 
[29] C. Muscalu and W. Schlag. Classical and multilinear harmonic analysis. Vol. I, volume 137 of Cambridge Studies in Advanced Mathematics. Cambridge University Press, Cambridge, 2013.

[30] F. Planchon. On uniqueness for semilinear wave equations. Math. Z., 244(3):587-599, 2003.

[31] G. Ponce and T. C. Sideris. Local regularity of nonlinear wave equations in three space dimensions. Comm. Partial Differential Equations, 18(1-2):169-177, 1993.

[32] M. A. Rammaha. A note on a nonlinear wave equation in two and three space dimensions. Comm. Partial Differential Equations, 22 (1997), no. 5-6, 799-810.

[33] T. C. Sideris. Global behavior of solutions to nonlinear wave equations in three dimensions. Comm. Partial Differential Equations, 8(12):1291-1323, 1983.

[34] H. F. Smith, C. D. Sogge, and C. Wang. Strichartz estimates for Dirichlet-wave equations in two dimensions with applications. Trans. Amer. Math. Soc., 364(6):3329-3347, 2012.

[35] H. F. Smith and D. Tataru. Sharp local well-posedness results for the nonlinear wave equation. Ann. of Math. (2), 162(1):291-366, 2005.

[36] G. Staffilani. On the generalized Korteweg-de Vries-type equations. Differential Integral Equations, 10(4):777-796, 1997.

[37] J. Sterbenz. Angular regularity and Strichartz estimates for the wave equation. Int. Math. Res. Not., (4):187-231, 2005. With an appendix by Igor Rodnianski.

[38] J. Sterbenz. Global regularity and scattering for general non-linear wave equations. II. $(4+1)$ dimensional Yang-Mills equations in the Lorentz gauge. Amer. J. Math., 129(3):611-664, 2007.

[39] D. Tataru. On the equation $\square u=|\nabla u|^{2}$ in 5+1 dimensions. Math. Res. Lett., 6(5-6):469-485, 1999.

[40] M. E. Taylor. Tools for PDE, volume 81 of Mathematical Surveys and Monographs. American Mathematical Society, Providence, RI, 2000. Pseudodifferential operators, paradifferential operators, and layer potentials.

[41] N. Tzvetkov. Existence of global solutions to nonlinear massless Dirac system and wave equation with small data. Tsukuba J. Math., 22(1):193-211, 1998.

[42] C. Wang. The Glassey conjecture on asymptotically flat manifolds. Trans. Amer. Math. Soc., 367(10):7429-7451, 2015.

[43] Y. Zhou. Uniqueness of generalized solutions to nonlinear wave equations. Amer. J. Math., 122(5):939-965, 2000.

[44] Y. Zhou. Blow up of solutions to the Cauchy problem for nonlinear wave equations. Chinese Ann. Math. Ser. B, 22(3):275-280, 2001.

Department of Mathematics, Faculty of Education, Mie University, 1577 KurimaMACHIYA-CHO, TsU, MiE 514-8507, JAPAN

E-mail address: hidano@edu.mie-u.ac.jp

Department of Mathematics, National Tsing Hua University, Hsinchu, Taiwan 30013, R.O.C.

E-mail address: jcjiang@math.nthu.edu.tw

Department of Mathematical Sciences, Seoul National University, Seoul 151-747, REPUBLIC OF KOREA

E-mail address: shklee@snu.ac.kr

School of Mathematical Sciences, Zhejiang University, Hangzhou 310027, China

E-mail address: wangcbo@gmail.com

URL: http://www.math.zju.edu.cn/wang 